\title{
ARID1A mutation sensitizes most ovarian clear cell carcinomas to BET inhibitors
}

\author{
Katrien Berns ${ }^{1}$ - Joseph J. Caumanns ${ }^{2}$ - E. Marielle Hijmans ${ }^{1}$ - Annemiek M. C. Gennissen ${ }^{1} \cdot$ Tesa M. Severson ${ }^{1}$. \\ Bastiaan Evers $^{1}$ - G. Bea A. Wisman ${ }^{2}$ - Gert Jan Meersma ${ }^{2}$ - Cor Lieftink ${ }^{1}$ - Roderick L. Beijersbergen ${ }^{1}$. \\ Hiroaki Itamochi ${ }^{3} \cdot$ Ate G. J. van der Zee ${ }^{2} \cdot$ Steven de Jong ${ }^{4} \cdot$ René Bernards $\mathbb{D}^{1}$
}

Received: 27 November 2017 / Revised: 23 March 2018 / Accepted: 10 April 2018 / Published online: 15 May 2018

(c) The Author(s) 2018. This article is published with open access

\begin{abstract}
Current treatment for advanced stage ovarian clear cell cancer is severely hampered by a lack of effective systemic therapy options, leading to a poor outlook for these patients. Sequencing studies revealed that ARID1A is mutated in over $50 \%$ of ovarian clear cell carcinomas. To search for a rational approach to target ovarian clear cell cancers with ARIDIA mutations, we performed kinome-centered lethality screens in a large panel of ovarian clear cell carcinoma cell lines. Using the largest OCCC cell line panel established to date, we show here that BRD2 inhibition is predominantly lethal in ARID1A mutated ovarian clear cell cancer cells. Importantly, small molecule inhibitors of the BET (bromodomain and extra terminal domain) family of proteins, to which BRD2 belongs, specifically inhibit proliferation of ARIDIA mutated cell lines, both in vitro and in ovarian clear cell cancer xenografts and patient-derived xenograft models. BET inhibitors cause a reduction in the expression of multiple SWI/SNF members including ARIDIB, providing a potential explanation for the observed lethal interaction with ARIDIA loss. Our data indicate that BET inhibition may represent a novel treatment strategy for a subset of ARIDIA mutated ovarian clear cell carcinomas.
\end{abstract}

These authors contributed equally: Katrien Berns and Joseph J.

Caumanns

Electronic supplementary material The online version of this article (https://doi.org/10.1038/s41388-018-0300-6) contains supplementary material, which is available to authorized users.

Katrien Berns

k.berns@nki.nl

$\triangle$ René Bernards

r.bernards@nki.nl

1 Division of Molecular Carcinogenesis and Oncode Institute, The Netherlands Cancer Institute, Plesmanlaan 121, Amsterdam 1066 CX, The Netherlands

2 Gynaecologic Oncology, Cancer Research Centre Groningen, University Medical Center Groningen, University of Groningen, Hanzeplein 1, Groningen 9713 GZ, The Netherlands

3 Department of Obstetrics and Gynaecology, Iwate Medical University School of Medicine, Iwate, Morioka 020-8505, Japan

4 Medical Oncology, Cancer Research Centre Groningen, University Medical Center Groningen, University of Groningen, Hanzeplein 1, Groningen 9713 GZ, The Netherlands

\section{Introduction}

Epithelial ovarian cancer covers approximately $90 \%$ of all ovarian cancers and is the most common cause of mortality in women with gynecologic cancers. Five histological subtypes have been defined for epithelial ovarian cancers: high-grade serous, low-grade serous, clear cell, mucinous, and endometrioid [1,2]. Although each subtype has unique molecular and clinical features, all epithelial ovarian subtypes are still treated similarly, consisting of de-bulking surgery in combination with platinum-based chemotherapy. Ovarian clear cell carcinoma (OCCC), the second most common subtype, appears to have a worse prognosis than the more common high-grade serous carcinoma, suggesting that current treatments are ineffective for OCCC, especially related to a poor response to platinum-based chemotherapy. Therefore, new treatment strategies for OCCC are urgently needed [3]. Development of OCCC has been linked to endometriosis and is characterized by a high mutation frequency of ARIDIA (>50\%), a subunit of the SWI/SNF chromatin remodeling complex $[4,5]$. Given the nature of the mutations it is generally accepted that ARID1A functions as a tumor suppressor gene. The SWI/SNF chromatin- 


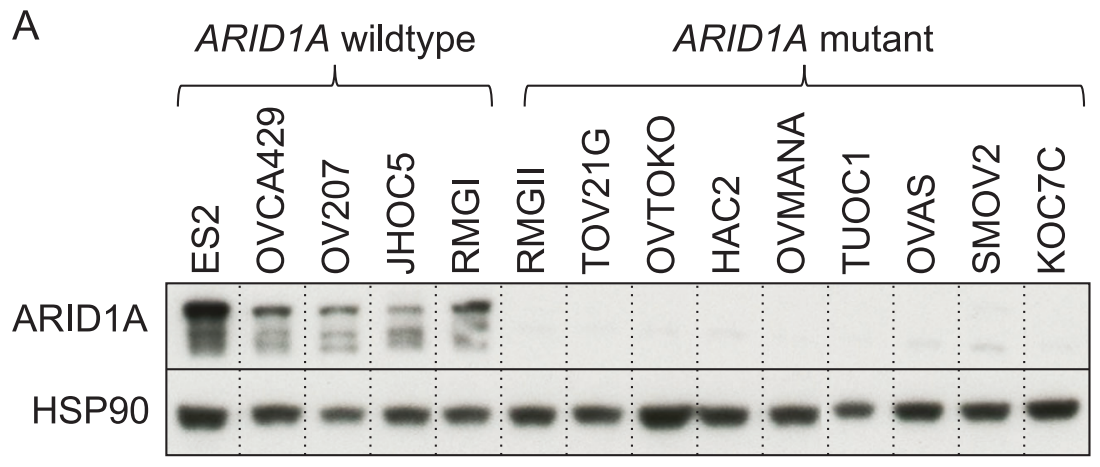

\section{$B$ infect TRC kinome}
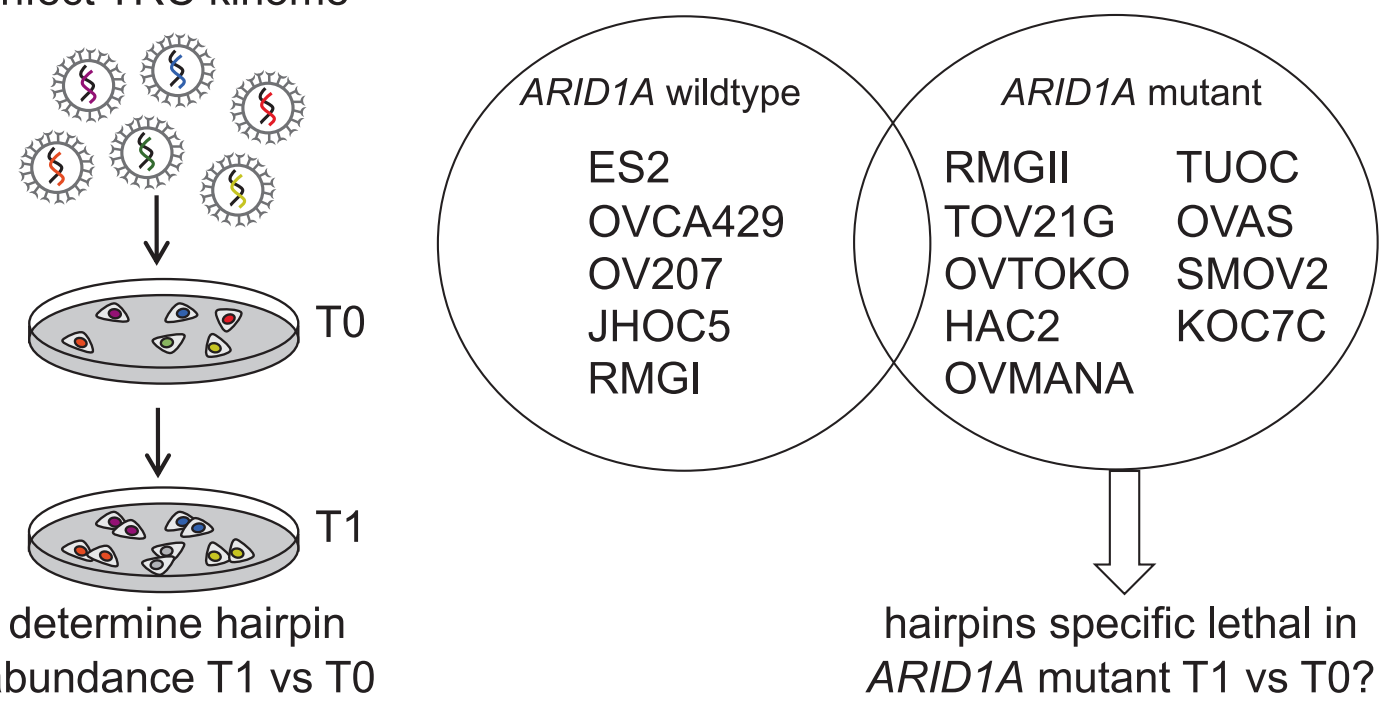

hairpins specific lethal in abundance T1 vs T0 ARID1A mutant T1 vs T0?

\begin{tabular}{|c|c|c|c|c|c|}
\hline \multicolumn{3}{|c|}{$A R I D 1 A$ specific lethal hits } & \multicolumn{3}{|c|}{ Common lethal hits } \\
\hline & ARID1A WT & ARID1A MUT & & ARID1A WT & ARID1A MUT \\
\hline$B R D 2$ & $0 / 5$ & $5 / 9$ & PLK1 & $5 / 5$ & $7 / 9$ \\
\hline PRPF4B & $0 / 5$ & $3 / 9$ & CHEK1 & $5 / 5$ & $4 / 9$ \\
\hline MYO3B & $1 / 5$ & $5 / 9$ & CDC2L1 & $4 / 5$ & $5 / 9$ \\
\hline PKN1 & $1 / 5$ & $4 / 9$ & TRRAP & $4 / 5$ & $5 / 9$ \\
\hline$P R K C Q$ & $1 / 5$ & $4 / 9$ & TAF1 & $4 / 5$ & $5 / 9$ \\
\hline
\end{tabular}

Fig. 1 TRC kinome screen in OCCC cell panel. a ARID1A protein expression levels of the OCCC panel were measured by Western blot analysis. HSP90 was used as a loading control. b Each cell line from the OCCC panel was infected in triplicate with the lentiviral TRC kinome library with a multiplicity of infection below 0.5 and with cell amounts ensuring $1000 \times$ coverage of the library. Upon stable selection of the library timepoint zero (TO) was collected, followed by culturing of the cells for an additional two weeks, after which timepoint one (T1) was harvested. The relative abundance of the hairpins in $T 0$ versus $T 1$

remodeling complex regulates the dynamic repositioning of nucleosomes, therefore the loss of ARIDIA could globally impact gene expression through deregulated transcription [6]. Since ARIDIA mutations have been identified in pre- was determined by deep sequencing. c Ranked lists for the lethal kinases were generated for every OCCC line based on three criteria (fold depletion, Geometric mean value of all hairpins and Second-best gene rank according to RIGER) as outlined in the methods section. From these ranked hit lists we determined which genes were lethal upon knockdown in all cell lines (common lethal hits) or preferentially in the ARIDIA mutant lines (ARIDIA specific lethal hits) and a top 5 is shown

neoplastic lesions it is suspected that ARID1A loss is an early event in the development of OCCC [4]. Possibly, loss of ARID1A activates major signaling pathways that confer an advantage to the tumor cells through enhanced 
A

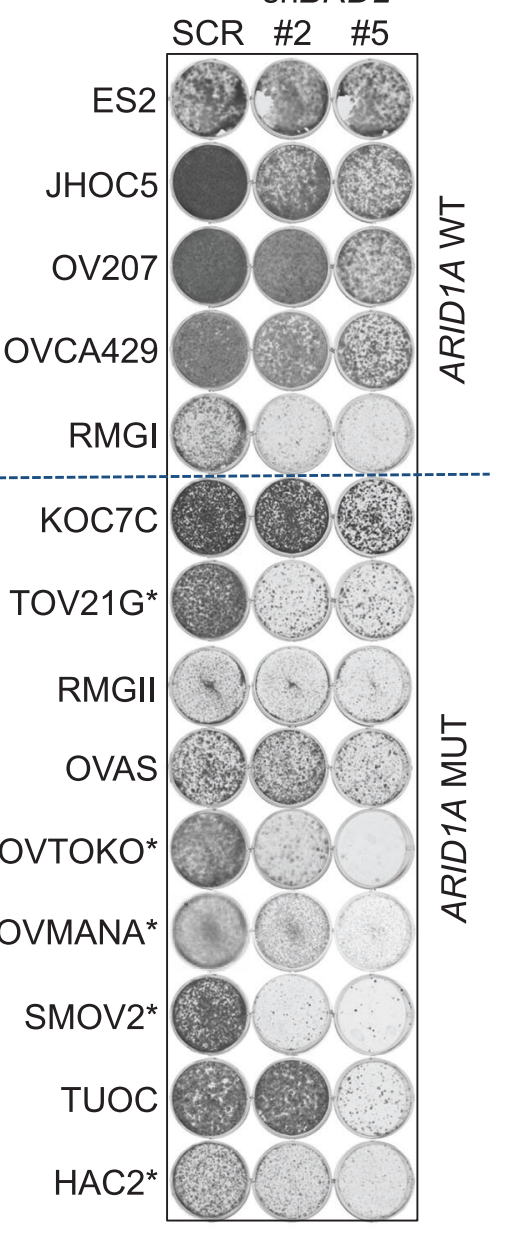

B

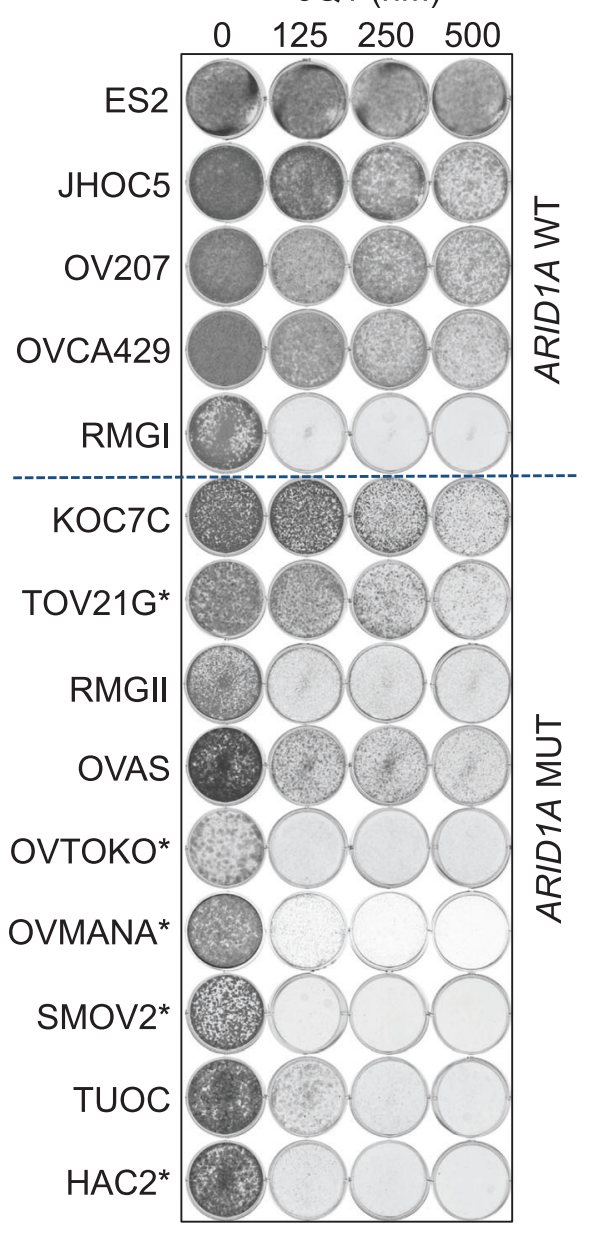

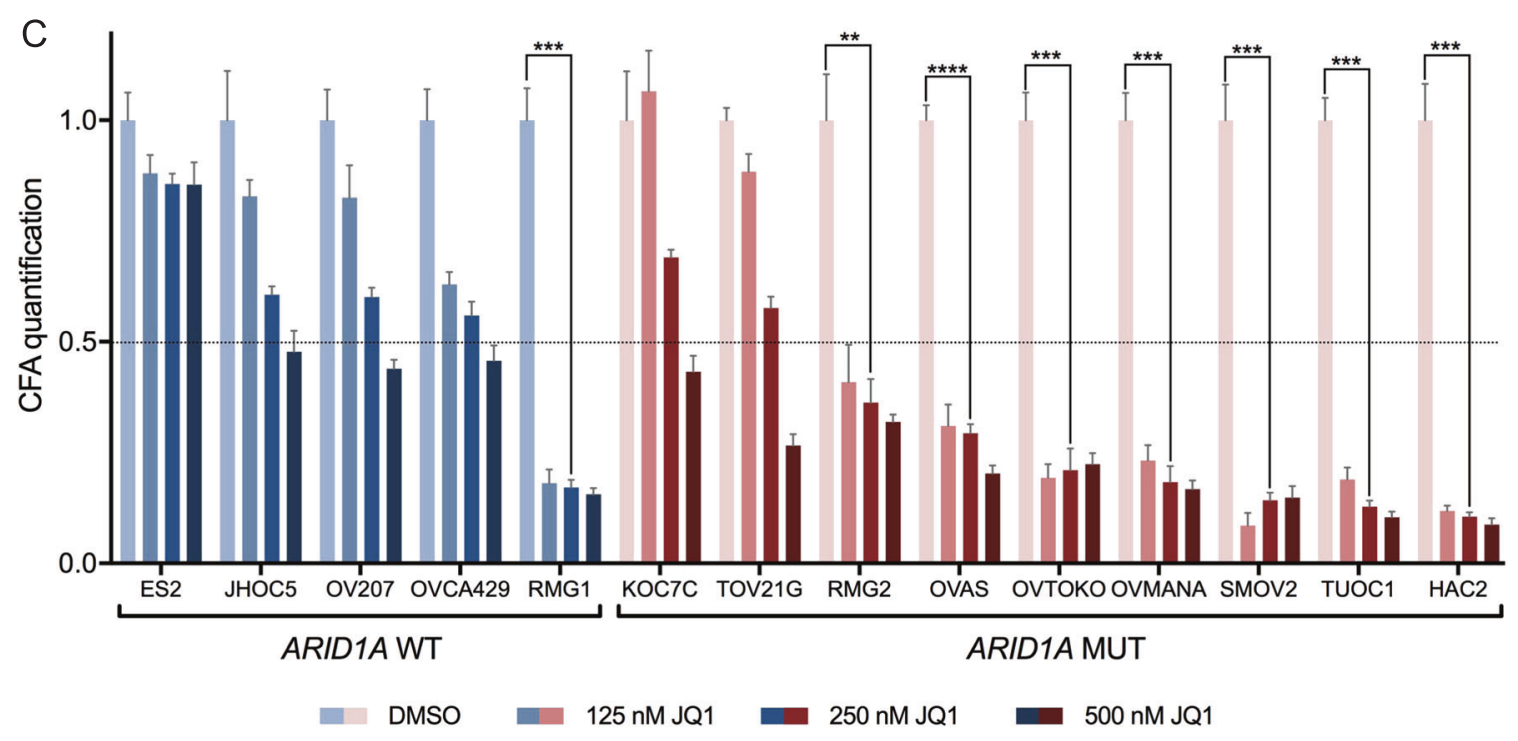

proliferation and/or survival. Given that ARIDIA is inactivated by mutation in OCCC, we pursued a synthetic lethal screening strategy to identify druggable targets in OCCC. We performed lethal kinome short hairpin (shRNA) screens in the largest panel of OCCC cell lines established to date having different ARIDIA mutation status. Here, we report the identification of $B R D 2$, a member of the BET (bromodomain and extra terminal domain) family, whose 
Fig. 2 BRD2 validates as ARID1A mutant specific lethal hit. a The functional phenotypes of non-overlapping lentiviral $\operatorname{sh} B R D 2$ vectors (\#2 and \#5) in the OCCC cell line panel were measured in a long-term colony formation assay. Cells expressing a mixture of nonfunctional scrambled hairpins (SCR) were used as control. The cells were fixed, stained and photographed after 10-12 days. In the cell lines labeled with an asterisk $(*) B R D 2$ was identified as lethal hit. $\mathbf{b}$ The indicated ARID1A wildtype and mutant OCCC cell lines were plated in 6-well plates $(10,000$ cells per well) and exposed to increasing JQ1 $(0,125$, 250 , and $500 \mathrm{nM}$ ) concentrations in triplicate. The cells were fixed, stained with crystal violet and photographed after 10 days. c Colony formation assays (CFA) from $\mathbf{b}$ were quantified by crystal violet dye extraction. Shown is relative reduction in crystal violet staining compared to untreated control. Colony formation assays were performed in triplicate. Error bars denote SD. The dotted line denotes the cut-off used (below 0.5 at $250 \mathrm{nM} \mathrm{JQ1} \mathrm{concentration)} \mathrm{to} \mathrm{qualify} \mathrm{cell}$ lines as "JQ1 sensitive". A Fisher Exact test with the abovementioned cutoff at $250 \mathrm{nM} \mathrm{JQ1}$ gave the statistic value of 0.09. $P$-values (only shown for the "JQ1 sensitive" lines) were calculated with multiple $t$ tests, asterisk denote the number of digits after the decimal

knockdown resulted in enhanced toxicity predominantly in ARIDIA mutant cell lines. Importantly, our data demonstrate enhanced sensitivity of ARIDIA mutated cells to the BET inhibitors JQ1 and iBET-762. Furthermore, the in vitro drug sensitivity data presented here were validated both in OCCC cell line xenografts and in OCCC patient-derived xenograft (PDX) models. Collectively, our data suggest a new treatment option for ARIDIA mutant OCCC that warrants clinical exploration.

\section{Results}

\section{ARID1A synthetic lethal screens in OCCC cell line panel}

To investigate which vulnerabilities exist in ARIDIA mutant OCCC lines we collected a very sizeable and unique set of OCCC cell lines and validated their ARID1A status by both ARIDIA sequencing and ARID1A protein expression analysis. We screened in total 14 OCCC cell lines (5 ARIDIA wildtype and 9 ARIDIA mutant, Fig. 1a) with a lentiviral shRNA library covering the human kinome to search for kinases whose suppression is specifically lethal in an ARIDIA mutant context. We have previously described kinome-centered screening approaches using this library [7, 8]. In short, each cell line was infected with the kinome shRNA library in triplicate, selected for presence of viral integration, cells were harvested at timepoint zero (T0) and the remainder was replated and cultured. After two to three weeks, cells were collected for timepoint one ( $T 1)$. Genomic DNA was isolated from both populations, and the relative abundance of the short hairpin sequences was determined by deep sequencing (Fig. 1b). For the selection of synthetic lethal genes, we set several criteria to ensure identification of hits with significant toxicity and with multiple hairpins (see Methods). Following these criteria, we generated ranked lists for the lethal kinases per cell line (Figure S1 and S2). By analyzing these cell-specific ranked lists we could identify common lethal kinases (PLK1, CHEK1, CDC2L1, TRRAP, and TAF1) and kinases that were more often lethal in the ARID1A mutant cell lines (BRD2, PRPF4B, MYO3B, $P K N 1$, and $P R K C Q$ ) (Fig. 1c). The lethal kinases that were exclusively selected in the ARID1A mutant lines (BRD2 and $P R P F 4 B$ ) were further validated. We subsequently observed that $P R P 4 F B$ loss was lethal in all the OCCC cell lines tested (data not shown), so toxicity appeared independent of ARIDIA mutation status. Interestingly, BRD2 depletion using two independent shRNA sequences (validated on protein levels Figure S3A) recapitulated the screening data more convincingly (Fig. 2a, Figure S3B). Especially in those ARIDIA mutant lines where BRD2 was previously identified as lethal hit (TOV21G, OVTOKO, HAC2, SMOV2, and OVMANA), significant killing with two $\operatorname{sh} B R D 2$ constructs was observed (with one $\operatorname{sh} B R D 2$ resulting in at least $70 \%$ cell number reduction) and $B R D 2$ was therefore considered as the only hit from our ARID1A synthetic-lethal screens. Validation of BRD2 depletion in the complete OCCC panel identified the cell line RMGI as one notable outlier, as BRD2 knockdown appeared to be toxic in this cell line whereas BRD2 was not identified as a lethal hit in the RMGI screen. Possibly, during validation $B R D 2$ shRNAs were introduced at higher titer compared to screening conditions leading to better knockdown and toxicity during validation procedure. Of note, we identified comparable BRD2 and BRD4 protein and RNA levels across the OCCC cell line panel indicating that different sensitivities towards BRD2 knockdown cannot be explained by the BRD2 and BRD4 status of the individual cell line (Figure S4A, B, C).

\section{BET domain inhibition specifically inhibits the proliferation of ARID1A mutant OCCC lines}

To further validate whether $B R D 2$ depletion caused proliferation defects predominantly in an ARIDIA mutant context, we targeted BRD2 function with the use of BET inhibitors. Selective inhibitors targeting BET proteins BRD2, BRD3, BRD4 as well as BRDT, have been described to exhibit antineoplastic activity [9]. BET inhibitors are currently in clinical trials for various hematological malignancies and solid tumors. BET inhibitors bind to acetylated lysine recognition motifs on the thereby preventing binding of BET proteins to the chromatin, resulting in disruption of subsequent chromatin remodeling and gene expression [10]. First, we tested the BET inhibitor JQ1 on our OCCC panel using a long-term proliferation assay. Interestingly, JQ1 sensitivity closely matched the BRD2 knockdown 
lethality across the OCCC cell line panel (Fig. 2a, b). Thus, ARIDIA mutant cells appear more sensitive to BET inhibition than ARIDIA wildtype cell lines. From the JQ1 colony formation assays we conclude that, based on a cutoff of $50 \%$ growth reduction at $250 \mathrm{nM} \mathrm{JQ1,} 1$ out of 5 ARIDIA wildtype cell lines and 7 out of 9 ARIDIA mutant cell lines are sensitive to the BET inhibitor (Fig. 2c). Collectively, these findings demonstrate that $B R D 2$ knockdown lethality closely resembles JQ1 sensitivity in OCCC lines, and that ARIDIA mutant lines are most sensitive to BET inhibition.

\section{ARID1A depletion enhances sensitivity to JQ1}

The genetic heterogeneity of the OCCC cell line panel poses a possible limitation in determining a genotypespecific toxicity. To firmly establish ARIDIA mutation as a direct cause of enhanced BET inhibitor sensitivity, we chose to test our findings further in OCCC isogenic cell line pairs. For this, we generated ARIDIA knockout subclones from the ARID1A wildtype cell lines ES2 and OVCA429 using CRISPR/Cas9 targeting. In both a polyclonal ES2 cell line having significant ARID1A reduction as well as a full knockout ES2 single cell clone, we observed that loss of ARIDIA significantly sensitized to BET inhibition by JQ1 (Fig. 3a, b). Similarly, the ARIDIA knockout OVCA429 subclones acquired enhanced sensitivity to JQ1 inhibition (Fig. 3c, d). These findings demonstrate a direct causal link between loss of ARID1A function and sensitivity towards BET inhibitors. We noted that the ARIDIA knockout clones adapted upon prolonged culturing (within months) in such a way that they gradually lost their enhanced sensitivity towards JQ1 inhibition (data not shown). This gradual adaptation to a drug-tolerant state has been observed before in other cell models [11]. Although speculative, we hypothesize that tumor-derived ARIDIA mutant OCCC lines may have acquired additional genetic alterations causing a more stable phenotype compared to cell lines generated by CRISPR/Cas9 mediated ARIDIA manipulation.

\section{JQ1 and iBET762 have similar activity in ARID1A deficient OCCC lines}

To further validate our findings, we tested another BET inhibitor, iBET762. First, we compared the inhibitors JQ1 and iBET762 in a long-term proliferation assay in two ARIDIA wildtype (ES2 and OVCA429) and two ARIDIA mutant (SMOV2 and HAC2) OCCC lines. The ARIDIA mutation dependent toxicity of BET inhibition appeared extremely similar for JQ1 and iBET762 in these cell lines (Fig. 3e, f). Next, iBET762 was tested on both ES2 (Fig. 3g) and OVCA429 (Fig. 3h) wildtype and ARID1A knockdown clones. Knockdown efficiencies for two independent
shARIDIA constructs were checked at the protein level (Fig. $3 \mathrm{i}, \mathrm{j}$ ). Upon ARIDIA knockdown, both OCCC lines displayed increased iBET762 lethality in these experiments (Fig. 3g, h). Collectively, we conclude that BET inhibition by the addition of either JQ1 or iBET762 is more toxic in ARID1A mutant lines, further validating our BRD2 screen hit.

\section{BET inhibition directly downregulates ARID1B expression}

We next sought to investigate why ARIDIA deficient cells exhibit an enhanced sensitivity towards BET inhibition. BET inhibitors likely exert a broad effect on transcription. A recent study identified $A R I D 1 B$, a SWI/SNF member mutually exclusive with ARIDIA in this complex, as a gene critically required for the survival of ARIDIA mutant cell lines [12]. Based on this we tested whether BET inhibition had an impact on ARID1B expression. First, we tested the effect of increasing amounts of JQ1 on ARIDIB expression in the OVCA429 ARIDIA wildtype and knockout cell lines. The differential sensitivity of these isogenic lines towards JQ1 was already demonstrated in Fig. 3. We indeed observed a concentration-dependent downregulation of $A R I D I B$ expression both at RNA (Fig. 4a) and protein levels (Fig. 4b). MYC protein levels were included as a positive control for effective inhibition. Similar results were obtained in the ES2 ARID1A wildtype and knockout cell lines (data not shown). Second, we observed that $B R D 2$ knockdown resulted in reduced ARID1B protein levels, directly implicating BRD2 in the regulation of $A R I D 1 B$ expression (Fig. 4c). Moreover, after knockdown of $A R I D I B$ expression in several OCCC lines, we observed toxicity only in an ARIDIA mutant background, in agreement with earlier published experiments (Fig. 4d, e). Of note, when testing the ARIDIA mutant lines in which BRD2 was not identified as a hit (KOC7C, OVAS, RMGII and TUOC1), we observed significant toxicity of ARIDIB knockdown in those lines that also displayed significant BETi toxicity (OVAS, RMGII, and TUOC1), suggesting that $B R D 2$ was probably not identified as a hit due to our hit-selection criteria or technical variations during screening procedures (Figure S4D,E). We noted that exogenous $A R I D 1 B$ overexpression could not rescue JQ1 mediated growth inhibition (data not shown) suggesting that multiple factors may be involved in JQ1 mediated cell toxicity. In accordance with previous studies [13], we noticed significant toxicity upon ARIDIB overexpression, which complicated these rescue experiments considerably. Collectively, our findings suggest that down-modulation of $A R I D 1 B$ by BET inhibitors contribute to the ARIDIA mutant specific vulnerability in OCCC cell lines, but likely is not solely responsible for the observed growth defects. 
A

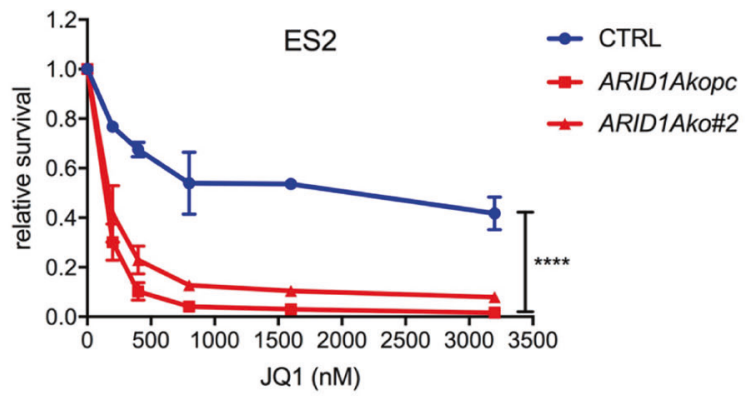

B

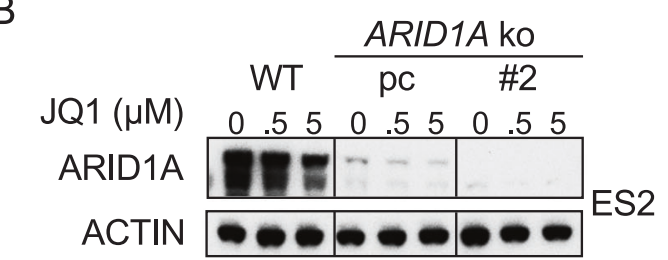

E
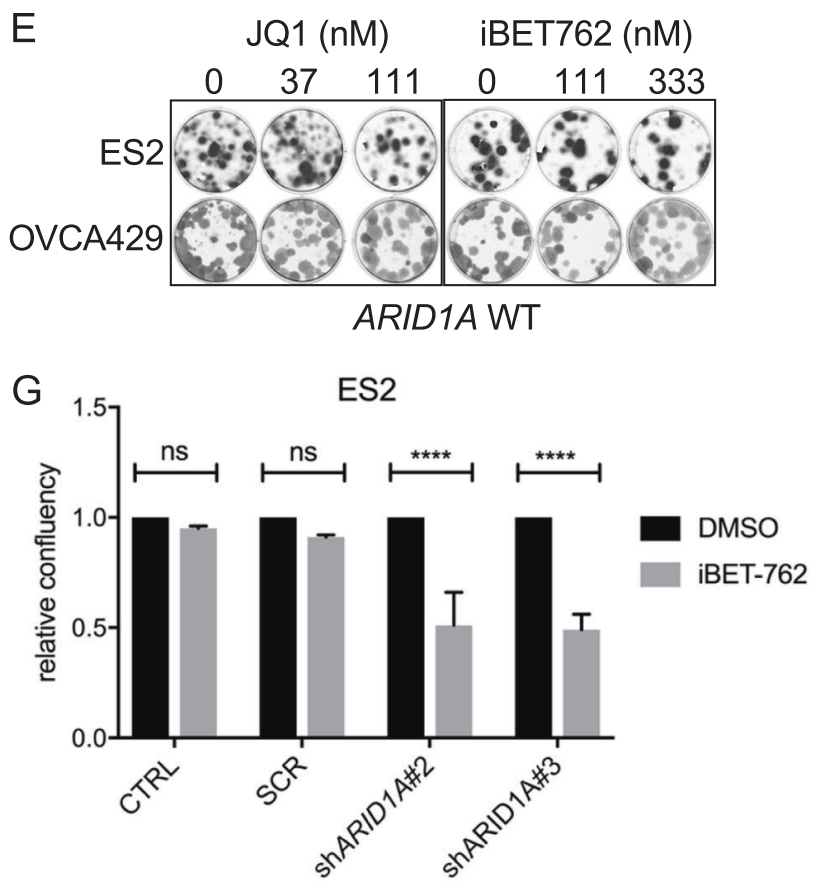

I

$\operatorname{sh} A R I D 1 A$

CTRL \#2 \#3

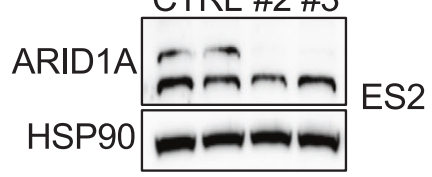

\section{BET domain inhibition inhibits expression of other SWI/SNF members}

When analyzing RNAseq data from OVCA429 ARID1A wildtype and knockout cells exposed to JQ1, we observed
C

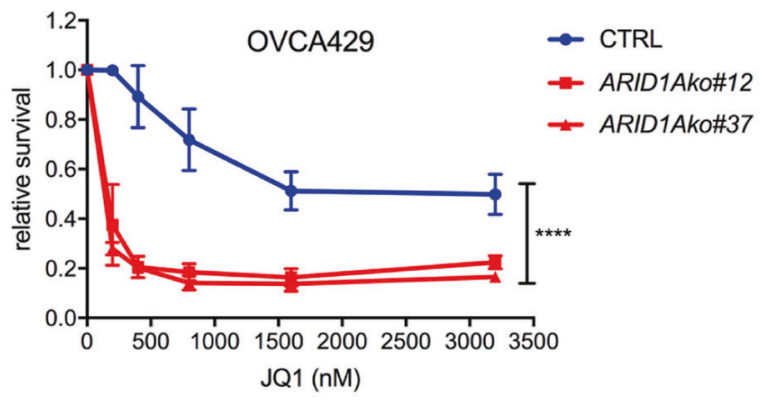

D

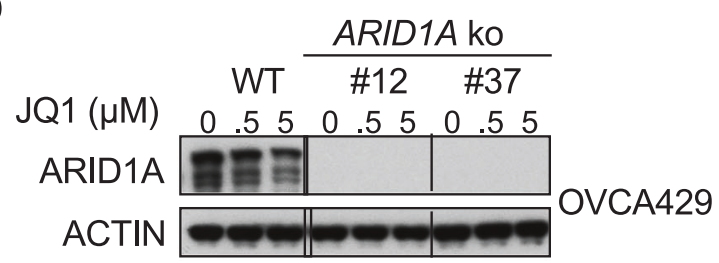

F

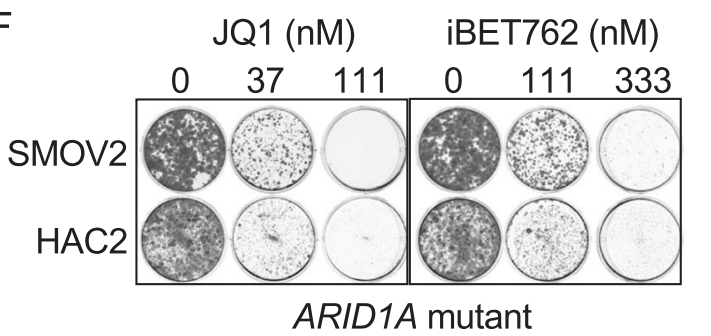

$\mathrm{H}$
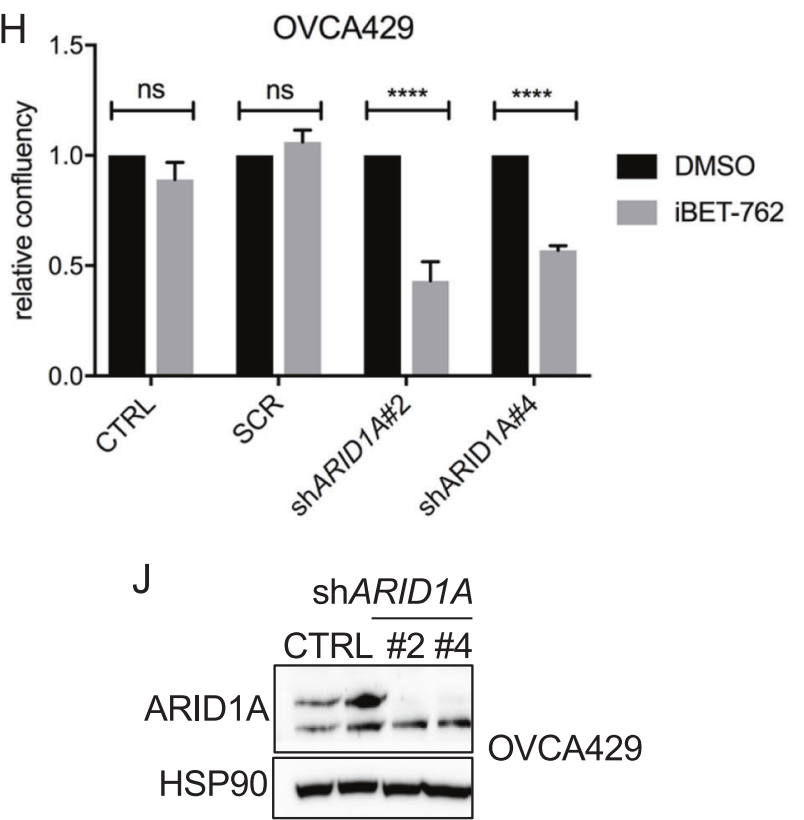

that BET inhibition resulted in transcriptional repression of several SWI/SNF family members besides ARIDIB, including SMARCC2 and SMARCE1 (data not shown). As such, BET inhibition may interfere with SWI/SNF function by affecting the transcription of multiple components of this 
Fig. 3 ARIDIA loss induces sensitivity towards BET inhibitors. a, c ES2 and OVCA429 ARID1A knockout cell lines were generated with a dual vector doxycycline inducible CRISPR/Cas9 vector system. For ES2, wildtype (wt) cells, one polyclonal knockout line (ARID1A kopc) and one monoclonal knockout line (ARID1A ko\#2) and for OVCA429, wt cells and two monoclonal knockout lines (ARID1A ko\#12 and \#37) were tested for JQ1 sensitivity in a 384 well 6-day cell viability assay using CellTiter Blue (CTB) as a readout. CTB measurements were normalized to untreated controls. Error bars denote SD. $P$-values were calculated with 2-way ANOVA, asterisk denote the number of digits after the decimal. b, d ARID1A Western blot analysis of ARID1A protein levels in the ES2 polyclonal ARID1A knockout clone and ES2/OVCA429 monoclonal ARID1A knockout clones. e, f The indicated two ARIDIA wildtype and ARIDIA mutant lines were exposed to increasing doses of BET inhibitors JQ1 and iBET762. After 10-12 days cells were stained and photographed. g, h ES2 and OVCA429 cell lines were stably infected with the indicated shRNA constructs targeting ARID1A and plated in 384 well plates. Confluency was monitored in the absence and presence of the BET inhibitor iBET762 $(800 \mathrm{nM})$. Shown are Incucyte confluency measurements relative to untreated cells from three independent experiments. Error bars denote SD. $P$-values were calculated with multiple t-tests, asterisk denote the number of digits after the decimal. i, j Western blots were performed on the ES2 and OVCA429 cell lines stably infected with the indicated shARIDIA constructs to monitor efficiency of ARID1A knockdown. HSP90 served as a loading control

multi-protein complex. To examine the generality of this observation we tested the effect of JQ1 exposure on gene expression of the SWI/SNF members ARIDIB, SMARCC2 and SMARCE1 in the complete OCCC panel. MYC was taken along as a control. Indeed, JQ1 treatment of the complete OCCC cell line panel resulted in a robust downregulation of the indicated SWI/SNF RNA levels, demonstrating a general effect of BET inhibition on the gene expression of SWI/SNF members (Fig. 5a, b). Notably, MYC RNA levels were downregulated by JQ1 exposure only in a subset of OCCC cell lines, suggesting that MYC repression by bromodomain inhibition is not a general phenomenon in OCCC cell lines.

Interestingly, chromatin immunoprecipitation experiments demonstrated specific BRD2 binding to various SWI/ SNF member promoter regions, including ARIDIA, ARIDIB, SMARCE1, and SMARCC2, both in ARIDIA wildtype (OVCA429) and ARIDIA mutant (HAC2) cell lines (Fig. 6a). Furthermore, JQ1 treatment efficiently inhibited BRD2 chromatin binding in OVCA429 and HAC2 (Fig. 6b, c, Figure S5). The BRD2 ChIP sequencing data were verified by qPCR of the $A R I D I B$ promoter region (Fig. 6d). Of note, in this experiment we also included a BRD4 ChIP and observed no binding of BRD4 to the same $A R I D 1 B$ promoter region (Fig. 6e). These observations suggest that the effects of BRD2 inhibition on ARID1B expression and other SWI/SNF members are specific and direct.

\section{In vivo OCCC models demonstrate ARID1A dependent sensitivity to JQ1}

To test the ARIDIA dependent sensitivity towards BET inhibition in vivo, we used NSG mice xenografted with OCCC cell lines. Unfortunately, engraftment efficiency of OCCC cells appeared rather low since from the tested ES2, SMOV2, TUOC1, and HAC2 cell lines, only ES2 and SMOV2 grew in mice. For the ARIDIA wildtype ES2 xenograft cohorts, we did not observe a significant growth difference between the vehicle and JQ1 treated tumors (Fig. 7a). In contrast, the ARID1A mutant SMOV2 xenografts were significantly growth impaired upon JQ1 treatment (Fig. 7b). In agreement with this, the tumor weights of excised tumors were not significantly reduced in JQ1 treated ES2 xenografts (Fig. 7c) but significantly declined in the JQ1 treated SMOV2 xenografts (Fig. 7d). Furthermore, Western blot analysis of SMOV2 tumor lysates demonstrated a significant reduction of ARID1B expression upon JQ1 treatment compared to DMSO vehicle control (Figure S6A). Thus, upon prolonged exposure in an in vivo model, BET inhibition downregulates ARID1B, likely contributing to the enhanced sensitivity of these tumor xenografts to JQ1. Given the low engraftment efficiencies of the OCCC cell lines, we reasoned that OCCC PDX models could provide an alternative strategy to further validate our results in vivo. $\mathrm{F} 3$ tumor pieces from an ARIDIA wildtype and an ARIDIA mutant (homozygous ARID1A 1148* stop-gained mutation) PDX model (Figure S6B), were subcutaneously implanted in NSG mice, and were randomized into vehicle control and JQ1 treatment groups. For the PDX-ARIDIAwildtype cohort, JQ1 treatment did not significantly impair tumor growth compared to vehicle control treatment (Fig. 7e). Importantly, tumor growth in the PDX-ARIDIA-mutant cohort was greatly impaired by JQ1 treatment (Fig. 7f). Thus, in agreement with the OCCC cell line xenograft experiments, ARIDIA mutant PDX tumors are sensitive to JQ1, whereas ARID1A wildtype PDX tumors are unaffected by JQ1 treatment. Of note, Ki67 staining was stronger reduced upon JQ1 treatment in the ARIDIA mutant PDX tumors, whereas Cleaved Caspase3 staining of the JQ1 treated tumors revealed a small but significant increase in apoptotic cells in the ARIDIA mutant PDX tumors (Figure S6C, D). Collectively, these observations support the notion that BET inhibitors are potentially useful for the treatment of ARIDIA mutant OCCC.

\section{Discussion}

Through loss of function genetic screens in a large panel of OCCC cell lines, we identify BRD2 loss as an ARIDIA mutation-specific dependency in OCCC cell lines. BRD2 is 

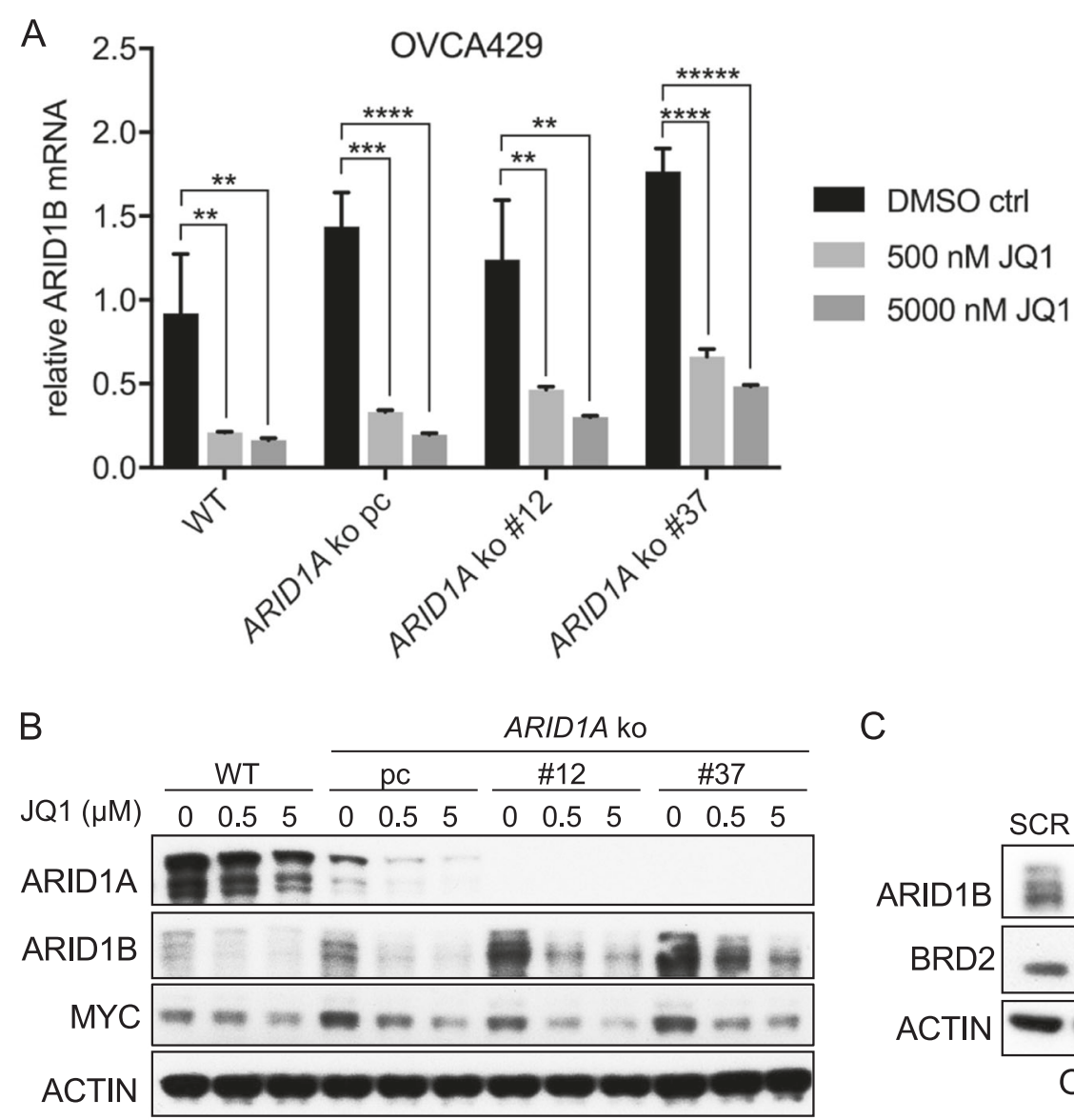

C

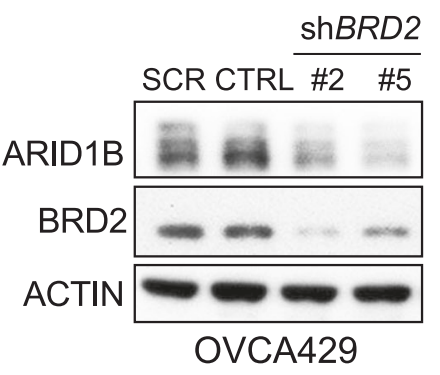

OVCA429

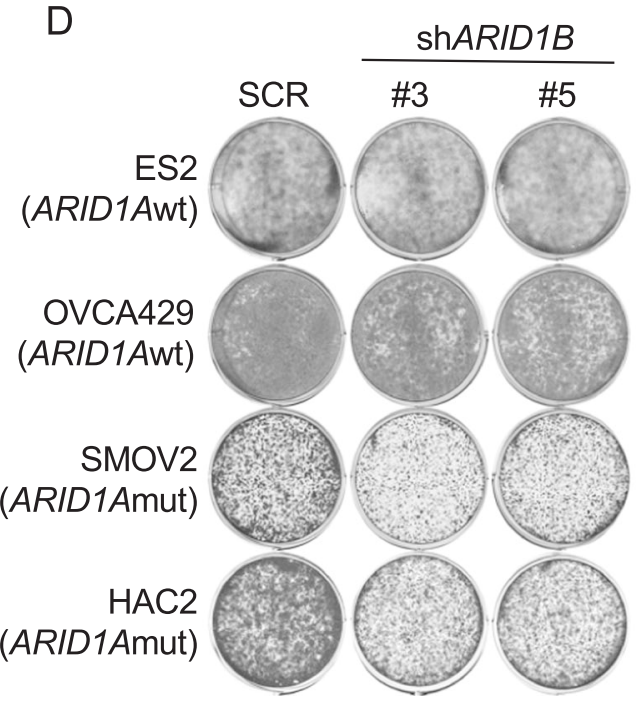

a member of the BET family of proteins consisting of BRD2, BRD3, BRD4, and BRDT. Bromodomain proteins are epigenetic readers, that play a role in transcriptional regulation through binding of hyperacetylated chromatin. BET proteins have also been reported to act as protein kinases, explaining their presence in the kinome library
E

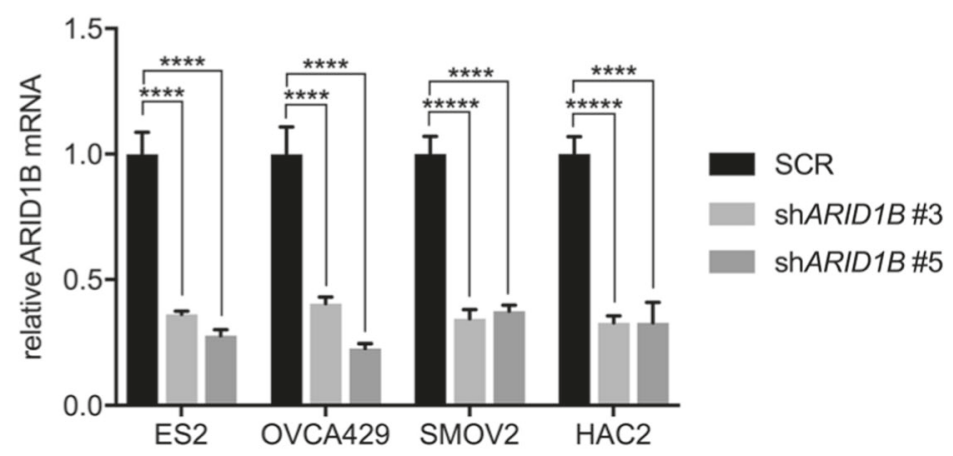

[14]. Importantly, inhibiting BRD2 function resulted in enhanced sensitivity of the ARIDIA mutant OCCC lines. Of note, $B R D 4$ loss appeared to be toxic in various OCCC lines independent of ARIDIA status, whereas BRD3 and BRDT were not selected as lethal hits in our screens (Figs. S1,2). Presumably, the BET family members exert diverse and 
Fig. 4 BET inhibition reduces ARID1B levels. a OVCA429 ARID1A knockout cell lines were generated with a dual vector doxycycline inducible CRISPR/Cas9 vector system. Wildtype cells, one polyclonal knockout line (ARIDIA kopc) and two monoclonal knockout lines (ARID1A ko\#12 and \#37) were exposed to increasing amounts of JQ1 as indicated. After $48 \mathrm{~h}$ drug exposure $A R I D I B$ RNA analysis was performed. mRNA levels were normalized to expression of GAPDH. Error bars denote SD. $P$-values were calculated with multiple $t$-tests, asterisk denote the number of digits after the decimal. b The OVCA429 cells described in a were subjected to Western blot analysis for the indicated proteins. ACTIN served as a loading control. c Western blot analysis of ARID1B and BRD2 in OVCA429 cells stably infected with lentiviral shRNA vectors targeting $B R D 2$. ACTIN served as a loading control. d Doxycycline inducible lentiviral ARIDIB shRNA vectors \#3 and \#5 were introduced in two ARID1A wildtype and two ARIDIA mutant OCCC lines. After stable selection cells were plated in a long-term proliferation assay in the presence of doxycycline. Cells expressing a mixture of nonfunctional scrambled hairpins (SCR) were used as control. The cells were fixed, stained and photographed after 10-14 days. e ARIDIB mRNA expression analysis by qRT-PCR in ES2, OVCA429, SMOV2 and HAC2 cells stably expressing the two doxycycline-inducible $\operatorname{sh} A R I D 1 B$ vectors. mRNA levels were normalized to expression of GAPDH. Error bars denote SD. $P$-values were calculated with multiple $t$-tests, asterisk denote the number of digits after the decimal

only partially overlapping functions in OCCC cell lines causing only loss of the BET member BRD2 to be synthetic lethal with ARIDIA mutation. In line with this assumption, a recent study reported a very different genome-wide occupancy of BRD2 and BRD4, suggesting non-redundant genomic functions [15]. Our observation that specifically $\mathrm{BRD} 2$, and not BRD4, binds to the ARIDIB promoter region suggests that BRD2 knockdown may have different effects on residual SWI/SNF function in ARIDIA mutant OCCC lines than BRD4 knockdown. Furthermore, gene essentiality data generated for luminal breast cancer cell lines recently uncovered a BET-independent requirement for BRD4, demonstrating that BRD4 knockdown lethality not always coincides with JQ1 sensitivity [16]. Indeed, also in our OCCC cell lines BRD4 knockdown lethality could not predict JQ1 response. However, more detailed information on the inhibitory efficiencies of both JQ1 and iBET762 on the different bromodomain family members in OCCC lines will be required to support this hypothesis. Interestingly, we show that ARIDIA depletion directly sensitized OCCC lines to BET inhibition, further strengthening the notion that ARIDIA loss is critical to the observed phenotype. Currently, we have no indications that ARIDIA loss sensitizes other cancer types to BET inhibition, suggesting an OCCC-specific context dependency for the findings we report here.

Our data suggest that $A R I D I B$ transcriptional downmodulation by BET inhibition contributes to the ARIDIA mutant dependent toxicity. However, we cannot rule out that other (SWI/SNF) factors regulated by members of the BET domain family contribute to the observed phenotypes.
It has been stated previously that ARID1B may be a potential therapeutic target for ARIDIA mutant cancers [12]. Importantly, we here demonstrate that BET inhibition may provide a way to target ARID1B indirectly, which could be explored further therapeutically. In that light, it is encouraging that the ARIDIA dependent sensitivity to BET inhibitors was also observed in OCCC xenografted mice treated with the BET inhibitor JQ1. Unfortunately, more extensive in vivo cell line validation experiments were complicated by the low tumor-take rate of xenografted OCCC cell lines. Therefore, the OCCC PDX experiments reported here provide important additional evidence that BET inhibition imposes an ARIDIA mutant dependent toxicity in an in vivo model.

It has been reported that inhibition of the methyltransferase EZH2 may represent a novel treatment strategy for ARIDIA mutant cancers [17]. Furthermore, recent studies have demonstrated a specific sensitivity of ARIDIA mutant OCCC cells to either dasatinib [18] or to the HDAC6 inhibitor ACY1215 [19]. For future studies it will be of interest to test these different synthetic lethal interactions together with our present findings to compare their ability to specifically target ARIDIA mutant OCCC in vivo. Of note, the OCCC cell line panel used in our study to search for ARIDIA mutation dependency is the largest reported to date, thereby most closely reflecting the mutation spectrum (e.g., concurrent hotspot mutations in PIK3CA, KRAS) found in OCCC patients [20]. As might be expected from such a heterogeneous group of cell lines, there was not a perfect separation between ARIDIA mutant and wildtype cell lines in terms of BET inhibitor response. However, we believe that the remarkable sensitivity of the majority of ARIDIA mutant OCCC lines for the BET inhibitors warrants further (pre)-clinical exploration.

In summary, we report here an unexpected and new synthetic lethal interaction between BRD2 loss and ARIDIA mutation. We suggest that the inhibitory effects on residual SWI/SNF function, specifically via reduced $A R I D 1 B$ expression, may explain the enhanced sensitivity of ARIDIA mutant cells to BET inhibitors. Our data imply that patients with ARIDIA mutant OCCC may benefit from BET domain inhibitors added to their treatment regimen.

\section{Materials and methods}

\section{OCCC cell lines and genotyping}

TOV21G was obtained from ATCC; OVTOKO, RMGI, RMGII, OVMANA, HAC2 from JCRB Cell Bank; JHOC5 from RIKEN Cell Bank; OVCA429 from Cell Biolabs; TUOC1, OVAS, SMOV2, and KOC7C were kindly provided by Hiroaki Itamochi; ES2 was a kind gift from Els 

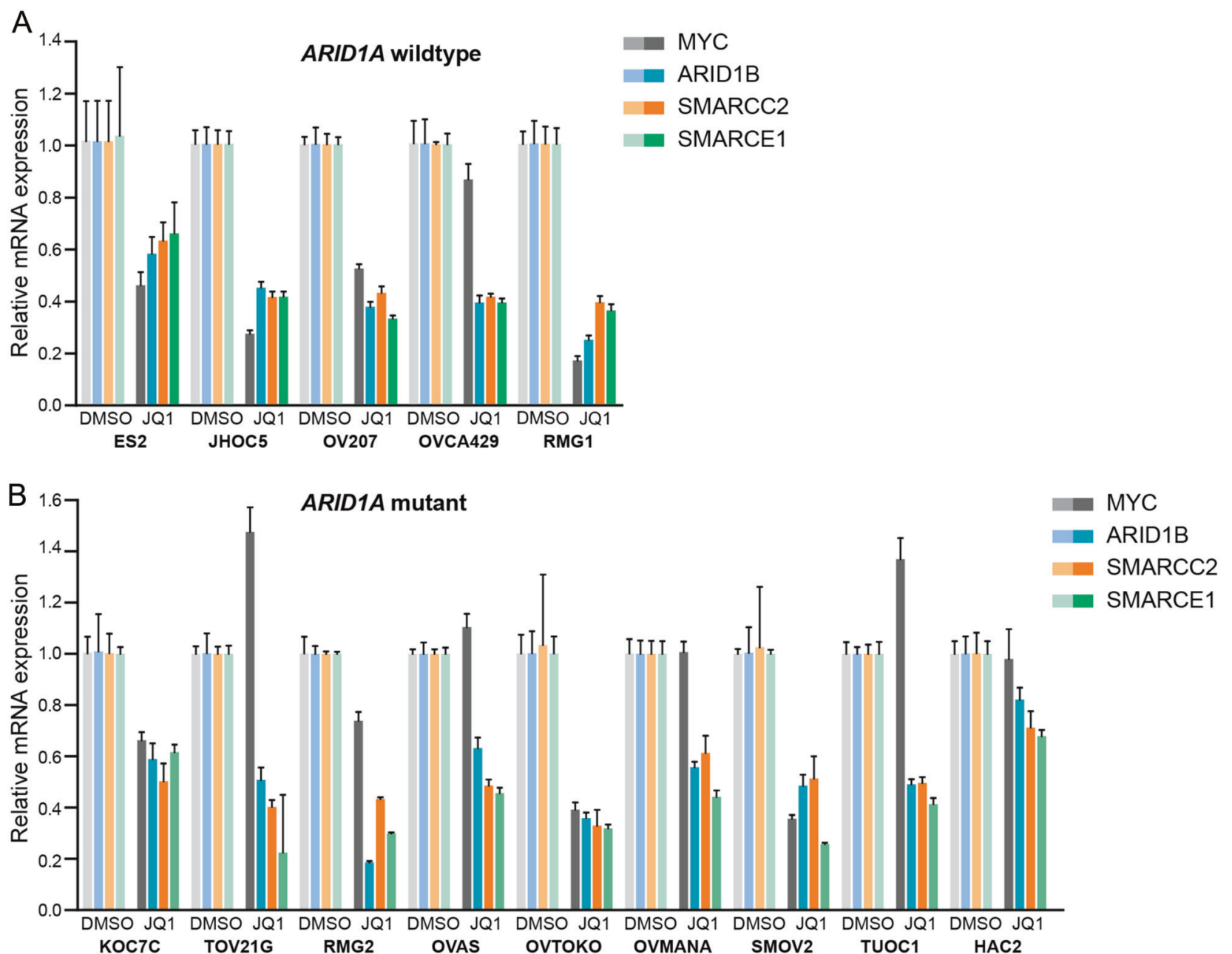

Fig. 5 BET inhibitors target multiple SWI/SNF members. a, b ARID1A wildtype (a) and ARID1A mutant (b) OCCC lines were exposed for $40 \mathrm{~h}$ to $500 \mathrm{nM}$ JQ1 and were subjected to mRNA

expression analysis with qRT-PCR. mRNA levels were normalized to expression of GAPDH and displayed is the relative expression of the indicated mRNAs to untreated cells. Error bars denote SD

Berns and OV207 was a kind gift from Vijayalakshmi Shridhar. All cells were maintained in RPMI supplemented with $10 \%$ Fetal Calf Serum and $100 \mu \mathrm{g} / \mathrm{ml}$ Penicilin/Streptomycin and $2 \mathrm{mM}$ L-Glutamine and tested negative for mycoplasma contamination. The Haloplex sequencing custom platform from Agilent was used to determine ARIDIA mutation status, with the target region design based on NM_139135 and NM_006015. OCCC cells were classified as "ARIDIA mutant" when homozygous frameshift and/ or nonsense ARIDIA mutations were detected in combination with no detectable ARID1A protein on Western.

\section{TRC kinome screen}

A kinome-centered short hairpin RNA (shRNA) library targeting 535 human kinases was assembled from the TRC human genome-wide shRNA collection (TRCHs1.0). Each OCCC line was stably transduced by lentiviral infection in triplicate with a multiplicity of infection (MOI) below 0.5 and sufficient number of cells to ensure a 1000-fold library coverage. A $T 0$ time point sample was taken from the cells stably expressing the shRNA library and the remainder of the cells was cultured for 2-3 weeks after which $T 1$ was harvested. The relative abundance of each shRNA comparing $T 1$ to $T 0$ was determined using the R/Bioconductor package DESeq [21]. Kinases were considered as lethal hits when the $P$ value calculated using DESeq was lower than 0.1 and the following three criteria were fulfilled: (1) at least one hairpin with a Fold Change below 0.3 (to ensure significant toxicity), (2) a geometric mean of all the hairpins below 0.8 (to ensure that the majority of hairpins have a similar effect, reducing falsepositive results), (3) a top 100 ranking according to the Second Best Gene Rank in RIGER (a method to rank genes by the rank of the second best scoring hairpin of that gene, ensuring selection of genes with at least two functional hairpins) [22]. The DESeq screening data for the 14 OCCC lines are enlisted in Supplementary Data file S1. 
A

BRD2 CHIP

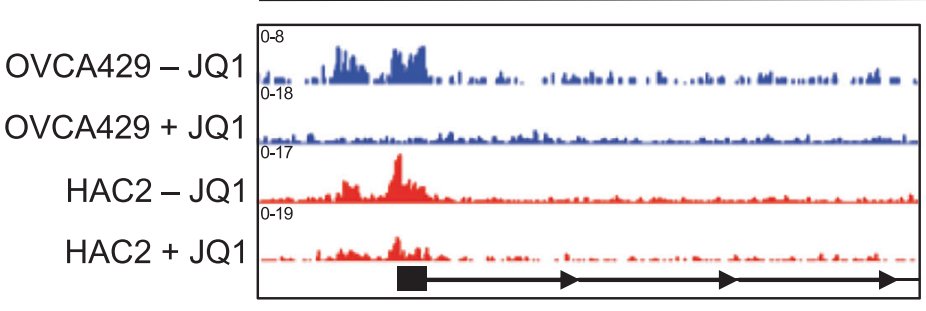

ARID1A

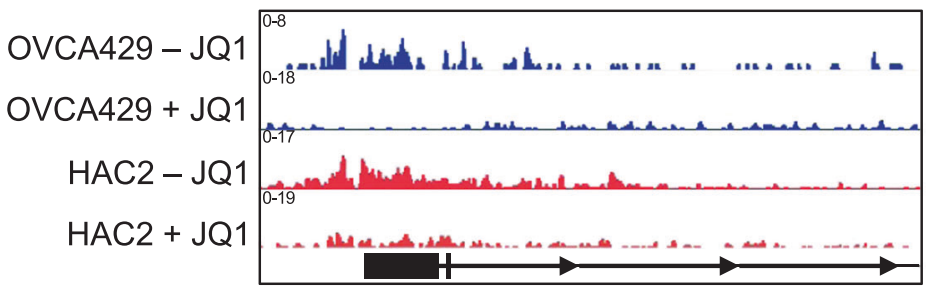

ARID1B

B

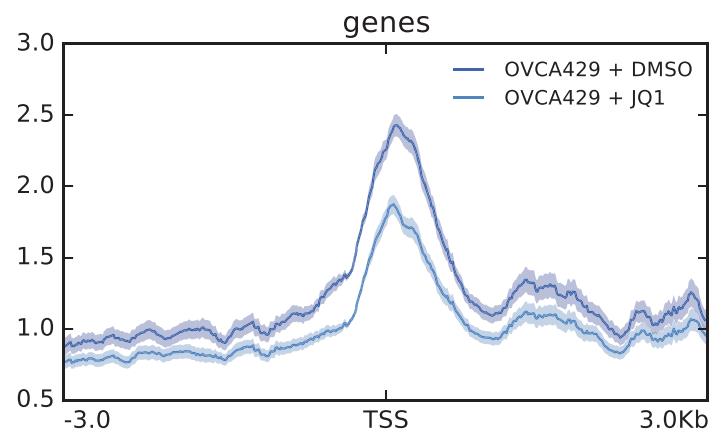

C

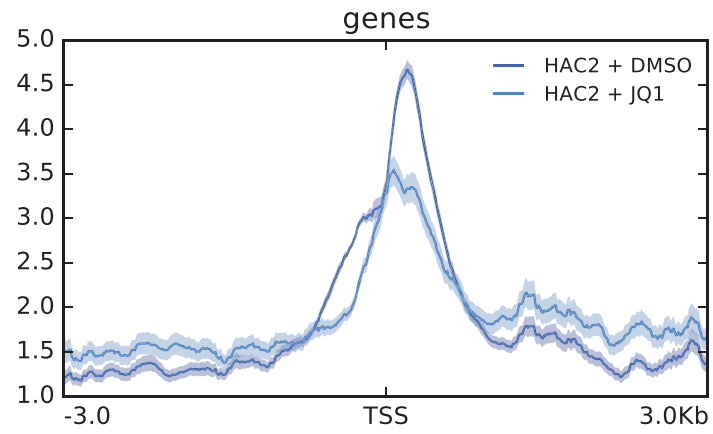

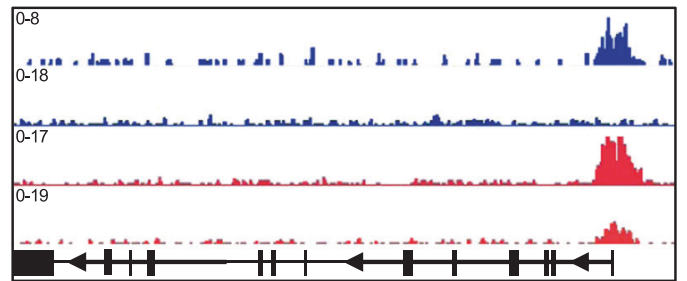

SMARCE1

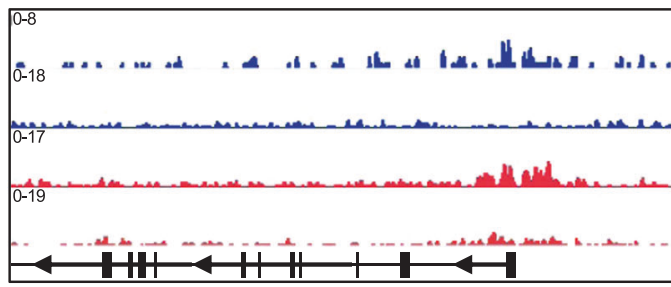

SMARCC2

D

$A R I D 1 B$

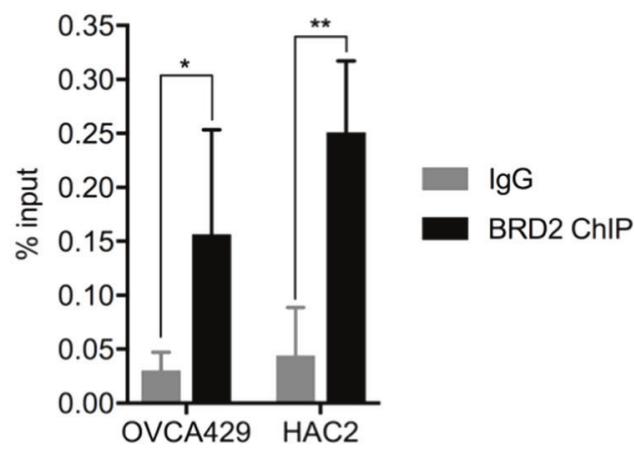

E $\quad A R I D 1 B$
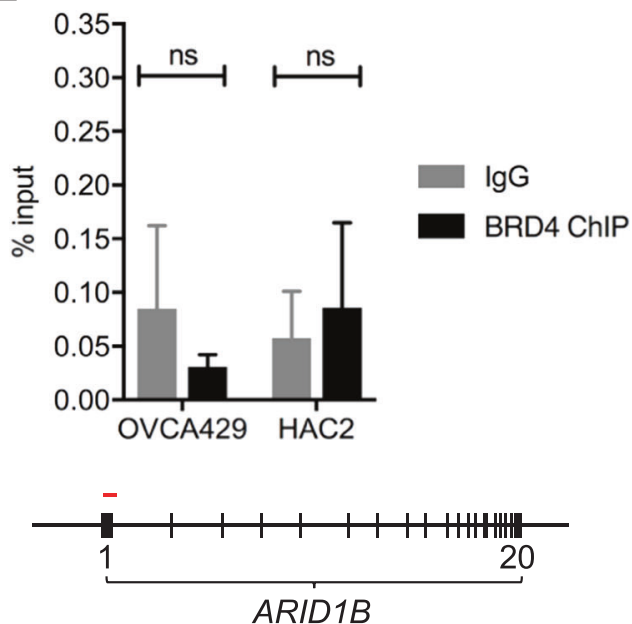

\section{Plasmids}

The following TRC pLKO.1 shRNA vectors were used for validation: BRD2\#2: TRCN0000006309; BRD2\#5:
TRCN0000006312. The lentiviral vector pLKO.1-Scramble shRNA was obtained from Addgene (\#1864). A doxycycline inducible lentiviral shRNA vector (GINSENG) [23] was modified as described [24] and was used to express the 
Fig. 6 Direct binding of BRD2 in ARID1B locus. a The ARID1A wildtype line OVCA429 and the ARID1A mutant line HAC2 were cultured for $24 \mathrm{~h}$ in $500 \mathrm{nM}$ JQ1 or DMSO vehicle control and subjected to chromatin immunoprecipitation with BRD2 and control antibodies. ChIP sequences were generated by Illumina Hiseq 2000 genome analyzer and aligned to the Human Reference Genome (assembly hg38) and visualized in IGV. Displayed are IGV snapshots of the Transcriptional Start Sites (TSS) of the indicated SWI/ SNF members ARID1A, ARID1B, SMARCE1 and SMARCC2. b, c Displayed are the average profiles of ChIP-seq signal for the indicated cell lines in absence and presence of JQ1 at transcriptional start site regions $(+/-3 \mathrm{~kb})(n=66035)$. Shading indicates standard error of average read count profiles. d, e qRTPCR amplification was performed with primers located in the $A R I D 1 B$ gene. Shown is the percentage of the BRD2 (d) and BRD4 (e) and control IgG chromatin immunoprecipitations over chromatin input qRT-PCR amplification. Error bars denote SD over $n=4$ (d) and $n=3$ (e) experiments. $P$-values were calculated with multiple t-tests, asterisk denote the number of digits after the decimal. Primer location is visualized in red in the gene map displayed underneath the graph

ARID1B hairpins (\#3: GGAAGATTAGAGGGTCACATA and \#5: GCCGAATTACAAACGCCATAT) under the control of doxycycline. ARIDIA knockout cell lines were generated with a dual vector doxycycline inducible CRISPR/Cas9 vector system (iKRUNC) as described [25] using the gRNA sequence targeting ARIDIA: AGGATGAGTCACGCCTCCAT. pLKO was used to express the ARIDIA shRNA hairpins with RNAi target sequences ARID1A\#2: AGTTGAAGTTCTGATGAA, ARIDIA\#3: GAGAAGTTGTATAGCACTA and ARIDIA\#4: GTGTAGACCCTTTCATGTA.

\section{Antibodies and compounds}

For Western blotting primary antibodies against ARID1A (PSG3), ACTIN (C2), HSP90 (H-114), CMYC (N-262), BRD4 (H-250) were obtained from Santa Cruz Biotechnology; BRD2 (A302-582A) from Bethyl; ARID1B (AB57461) and Histone-H3(trimethylK27) (AB6002) from Abcam. Immunohistochemical analysis of paraffinembedded xenograft slices were performed as described [26], using antibodies against Cleaved-Caspase3 from Cell Signaling (\#9661); Ki67 from DAKO (M7240) and ARID1A from Sigma (HPA005456). iBET762 was obtained from Xcess Biosciences. JQ1 for the cell line experiments was kindly provided by the Bradner lab (DanaFarber Cancer Institute, Boston, USA) [9] and later purchased from Axon Medchem (axon 1989); both batches had similar activity. JQ1 (HY-13030) used in the xenograft experiments was purchased from MedChem Express. Before use in animals, the in vitro activity of JQ1 (HY13030) was compared to the activity of JQ1 obtained from Axon Medchem.

\section{Quantitative RT-PCR}

The 7500 Fast Real-Time PCR System from Applied Biosystems was used to measure mRNA levels which were normalized to expression of GAPDH. Each QRT-PCR experiment included technical replicates and were repeated at least once. The following primer sequences were used in the SYBR ${ }^{\circledR}$ Green master mix (Roche): GAPDH_Forward, AAGGTGAAGGTCGGAGTCAA;

GAPDH_Reverse, AATGAAGGGGTCATTGATGG;

BRD2_Forward, GAGGTGTCCAATCCCAAAAAGC;

BRD2_Reverse, ATGCGAACTGATGTTTCCACA;

BRD4_Forward,

AATGAGCTACCCACAGAAGAAAC;

BRD4_Reverse, GAGTCGATGCTTGAGTTGTGTT;

ARIDIB_Forward, CAAGGGGATCAGAGCAACCC;

ARIDIB_Reverse, CTACCTGGGATACTTGCAGGA;

SMARCC2_Forward, TACTCTTGGGGGTTCAGTCG;

SMARCC2_Reverse, TCTTCAACGGCAAGAACAAG;

SMARCE1_Forward,

AACAACTACAGGCTGGGAGG;

SMARCE1_Reverse, CGGCTTATCTGGTGGCTTT.

\section{Proliferation assays}

For long-term colony formation assays, cells were cultured in 6-well plates and medium was refreshed every 3 days. After 10 days cells were fixed with $4 \%$ formaldehyde and stained with $0.1 \%$ crystal violet and subsequently scanned. Colony formation assay quantification was performed by optical density measurements of extracted dye at $590 \mathrm{nM}$. Proliferation assays were repeated at least three times, within one assay three technical replicates were performed. Representative stainings and quantifications are shown. Six-day growth assays were performed in quadruple in 384-well plates and were quantified with the cell viability assay CellTiter-Blue (Promega) or by confluency monitoring in an IncuCyte Zoom live cell imaging system (Essen BioScience). Measurements were normalized to untreated controls.

\section{Chromatin immunoprecipitation}

Chromatin Immunoprecipitation (ChIP) was performed as described [27]. For each ChIP $4 \mu \mathrm{g}$ anti-BRD2 (A302-583A) or anti-BRD4 (A301-985A) from Bethyl Laboratories and control Rabbit IgG SC-2027 from Santa Cruz Biotechnology were used. ChIP sequences were generated with the use of the Illumina Hiseq 2000 genome analyzer. Mapped reads were visualized in heatmaps and profiles using deepTools 2 v2.4.0 with the UCSC hg38 refGene coordinates. QPCR of the $A R I D 1 B$ region was performed with ARID1B1.1_Forward, CGCCCACAATGTGCTTTAACGG; ARID1B1.1_Reverse, AGGAAAAACCCACTCGCTTGTC. 
A

ES2 xenograft

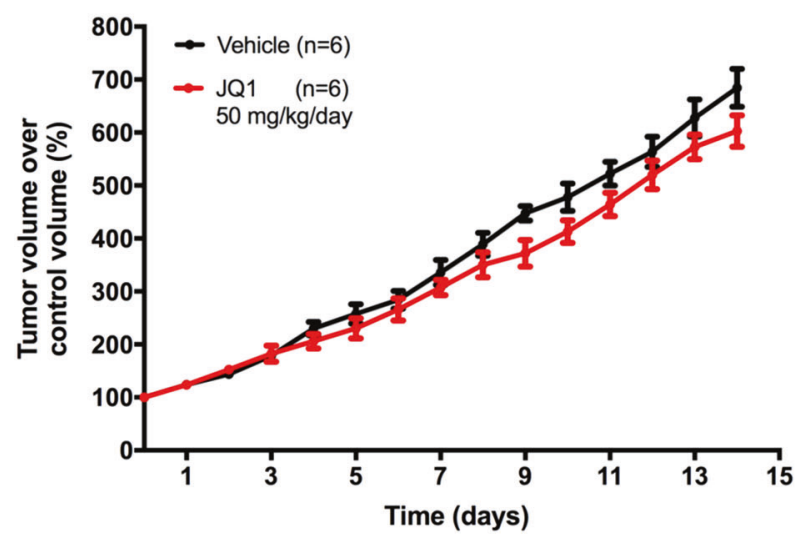

C

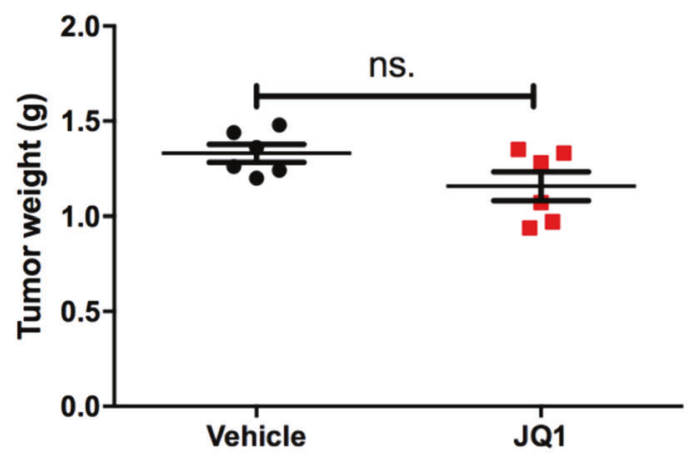

E

PDX - ARID1Awt

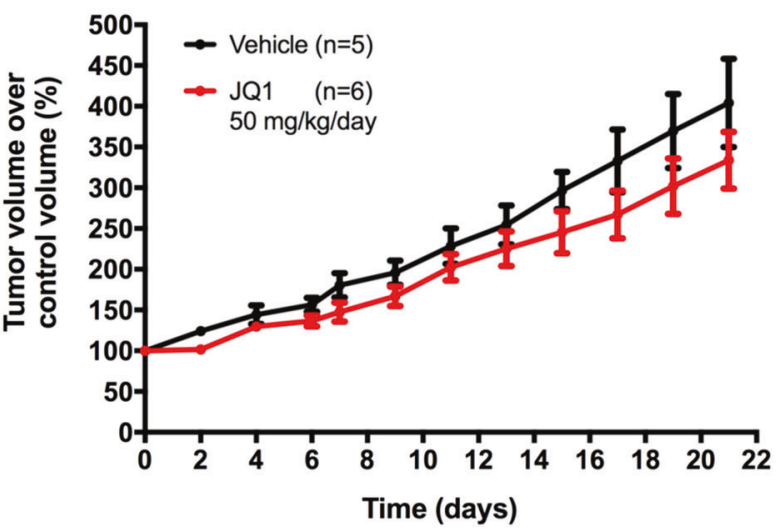

Fig. 7 JQ1 specifically inhibits in vivo growth of ARIDIA mutant OCCC xenografts and PDX models. a, b ARID1A wildtype ES2 cells $\left(5 \times 10^{6}\right.$ in PBS) were subcutaneously injected in the flank of 8-10weeks-old NSG mice. For the ARID1A mutant SMOV2 xenograft experiments, successfully established SMOV2 engraftments (see methods) were dissected, and tumor pieces were subcutaneously propagated in the flank of new mice. When tumors reached $\sim 200 \mathrm{~mm}^{3}$, mice were randomized into vehicle (DMSO) control or treatment (JQ1) groups ( $n=6 \mathrm{mice} /$ group). ES2 xenografts reached the $1500 \mathrm{~mm}^{3}$ endpoint after 14 days of JQ1 administration, SMOV2 xenografts were treated with JQ1 for 21 days. c, $\mathbf{d}$ Displayed are the tumorweights of the indicated excised tumors after vehicle or JQ1
B

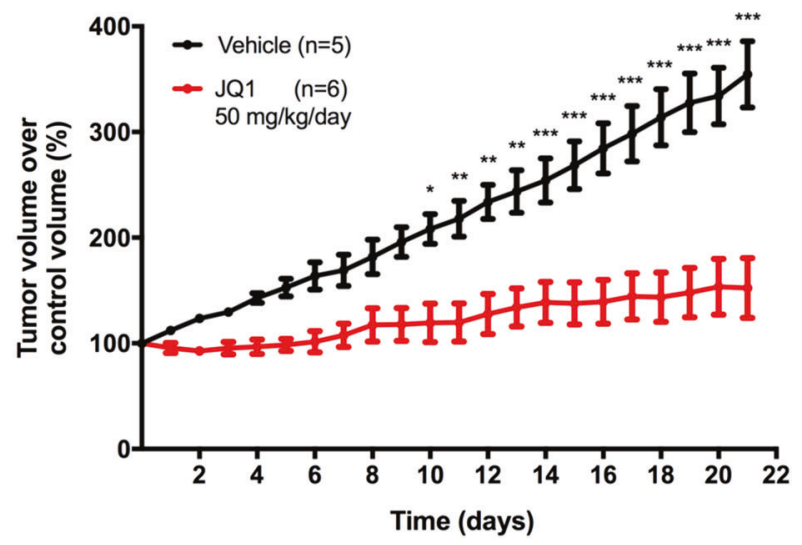

D SMOV2 Tumorweight

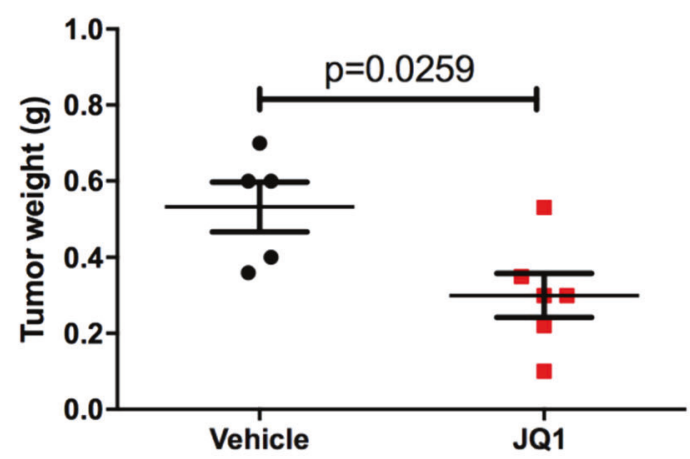

$\mathrm{F}$

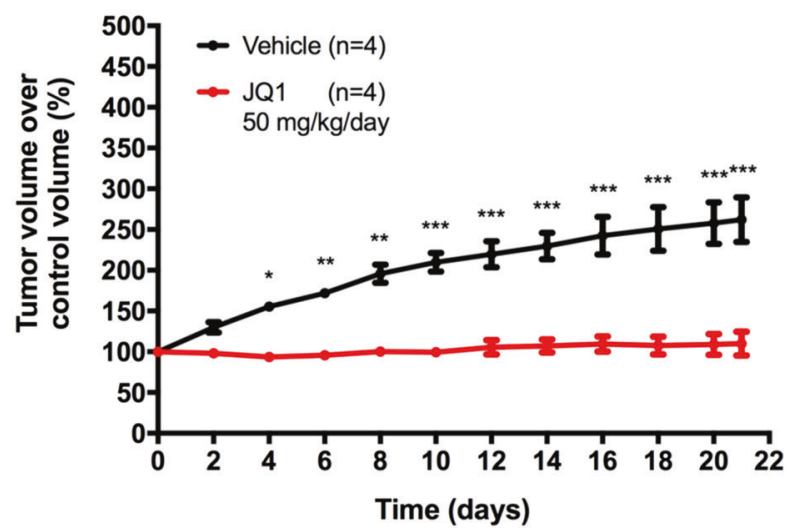

treatments. e, f ARID1A wildtype (e) and mutant (f) PDX F3 tumors were established (see methods) and tumor pieces were subcutaneously implanted in the flank of NSG mice. When tumors demonstrated sustained growth, mice were randomized into vehicle (DMSO) control or treatment (JQ1) groups. Treatment with JQ1 was continued for 21 days. For all in vivo experiments, JQ1 $(50 \mathrm{mg} / \mathrm{kg})$ or DMSO vehicle were administered intraperitoneally daily and tumor growth was quantified by caliper measurements. Tumor growth was determined as tumor volume treatment day/ tumor volume start treatment. Statistical significance for tumor growth was determined using twoway ANOVA with Bonferoni post-hoc test correction. Error bars denote standard error of mean 


\section{OCCC xenografts and PDX models}

All animal experiments were approved by the Institutional Animal Care and Use Committee of the University of Groningen and carried out in accordance with the approved guidelines. For the xenografts, ES2 cells $\left(5 \times 10^{6}\right.$ in PBS) and SMOV2 cells $\left(5 \times 10^{6}\right.$ in $50 \% \mathrm{PBS} / 50 \%$ Matrigel $)$ were subcutaneously injected in the flank of 8 to 10 weeks old NOD.CB17-Prkdcscid/NCrHsd (NSG) mice. Due to the long latency time (on average 75 days), we used successfully established SMOV2 xenografts for subsequent experiments. For this, SMOV2 xenografts were dissected, and $3 \times 3 \times 3 \mathrm{~mm}^{3}$ pieces were subcutaneously propagated in the flank of new mice. STR profiling was used to confirm SMOV2 identity of established xenografts. Thus, ES2 cells were injected and SMOV2 tumor pieces were transplanted in the flanks of NSG mice, and as soon as tumors reached the threshold size of $200 \mathrm{~mm}^{3}$, mice were randomized into vehicle control or treatment groups ( $n=6$ mice/group). JQ1 $(50 \mathrm{mg} / \mathrm{kg}$ in $10 \%$ DMSO, 9\% (2hydroxypropyl)- $\beta$-cyclodextrin) or vehicle (10\% DMSO, 9\% (2hydroxypropyl)- $\beta$ cyclodextrin) was daily administered intraperitoneally. Treatment with JQ1 was continued for 21 days as described in previous studies $[28,29]$. Based on the time to reach the humane endpoint for tumor size in mice $\left(\sim 1500 \mathrm{~mm}^{3}\right)$, ES2 tumor-bearing mice had to be euthanized after 14 days of JQ1 treatment.

OCCC PDX models were established as described previously [26]. Briefly, all patients gave written informed consent and tumor specimens were obtained during surgery. Clinical characteristics of the patient from which ARIDIA mutant OCCC PDX was established, were FIGO stage IIIC, no response to carboplatin/paclitaxel chemotherapy and a 9 months disease specific survival. ARIDIA wildtype PDX was established from an OCCC patient FIGO stage IIB that showed a full response to carboplatin/paclitaxel chemotherapy and no relapse after 13 months. OCCC PDX models were sequenced using Haloplex (Agilent technologies) to determine ARIDIA mutation status. F3 tumor pieces from an ARIDIA wildtype and ARIDIA mutant PDX model were cut into $3 \times 3 \times 3 \mathrm{~mm}^{3}$ pieces and subcutaneously implanted in NSG mice. When tumors demonstrated sustained growth (ARIDIA wildtype PDX on average 26 days, ARIDIA mutant PDX on average 34 days), mice were randomized into vehicle control or treatment groups $(n=5$ mice/group). JQ1 $(50 \mathrm{mg} / \mathrm{kg}$ in 10\% DMSO, 9\% (2hydroxypropyl)- $\beta$-cyclodextrin) or vehicle (10\% DMSO, 9\% (2hydroxypropyl)- $\beta$-cyclodextrin) was daily administered intraperitoneally. Treatment with JQ1 was continued for 21 days.

Sample size for mouse experiments were calculated to be four mice per group (using significance level alpha of 5\%, power $80 \%$, estimated effect in growth reduction $50 \%$ and coefficient variation of 25\%). 1-2 additional mice per group were added in case of dropouts. Animals were excluded when no initial tumor growth was detected before treatment start or animals had $>20 \%$ weight loss or died during treatment course. Variances between the groups being compared were similar.

Acknowledgements We thank pathologist E.W. Duiker from UMCG for her contributions on OCCC pathology, H. Maat (Department of Experimental Hematology, UMCG) for her help with ChIP experiments, the people from the Genomics Core Facility of the Netherlands Cancer Institute for their technical support, and members of the Bernards lab for their helpful discussions. This research was supported by grants from the Dutch Cancer Society (KWF, RUG2012-5477) and the Cancer Genomics Center Netherlands through the Netherlands Organization for Scientific Research (NWO).

\section{Compliance with ethical standards}

Conflict of interest The authors declare that they have no conflict of interest.

Open Access This article is licensed under a Creative Commons Attribution 4.0 International License, which permits use, sharing, adaptation, distribution and reproduction in any medium or format, as long as you give appropriate credit to the original author(s) and the source, provide a link to the Creative Commons license, and indicate if changes were made. The images or other third party material in this article are included in the article's Creative Commons license, unless indicated otherwise in a credit line to the material. If material is not included in the article's Creative Commons license and your intended use is not permitted by statutory regulation or exceeds the permitted use, you will need to obtain permission directly from the copyright holder. To view a copy of this license, visit http://creativecommons. org/licenses/by/4.0/.

\section{References}

1. Kurman RJ, Shih IM. Pathogenesis of ovarian cancer: lessons from morphology and molecular biology and their clinical implications. Int J Gynecol Pathol. 2008;27:151-60.

2. Prat J. New insights into ovarian cancer pathology. Ann Oncol. 2012;23:111-7.

3. Braicu EI, Sehouli J, Richter R, Pietzner K, Denkert C, Fotopoulou C. Role of histological type on surgical outcome and survival following radical primary tumour debulking of epithelial ovarian, fallopian tube and peritoneal cancers. $\mathrm{Br} \mathrm{J}$ Cancer. 2011;105:1818-24.

4. Wiegand KC, Shah SP, Al-Agha OM, Zhao Y, Tse K, Zeng T, et al. ARID1A mutations in endometriosis-associated ovarian carcinomas. New Engl J Med. 2010;363:1532-43.

5. Jones S,Wang TL,Shih Ie M,Mao TL, Nakayama K,Roden R, et al. Frequent mutations of chromatin remodeling gene ARID1A in ovarian clear cell carcinoma. Science. 2010;330:228-31.

6. Wilson BG, Roberts CW. SWI/SNF nucleosome remodellers and cancer. Nat Rev Cancer. 2011;11:481-92.

7. Sun C, Hobor S, Bertotti A, Zecchin D, Huang S, Galimi F, et al. Intrinsic resistance to MEK inhibition in KRAS mutant lung and colon cancer through transcriptional induction of ERBB3. Cell Rep. 2014;7:86-93.

8. Prahallad A, Sun C, Huang S, Di Nicolantonio F, Salazar R, Zecchin $\mathrm{D}$, et al. Unresponsiveness of colon cancer to BRAF 
(V600E) inhibition through feedback activation of EGFR. Nature. 2012;483:100-3.

9. Filippakopoulos P, Qi J, Picaud S, Shen Y, Smith WB, Fedorov $\mathrm{O}$, et al. Selective inhibition of BET bromodomains. Nature. 2010;468:1067-73.

10. Filippakopoulos P, Knapp S. Targeting bromodomains: epigenetic readers of lysine acetylation. Nat Rev Drug Discov. 2014;13:337-56.

11. Sharma SV, Lee DY, Li B, Quinlan MP, Takahashi F, Maheswaran S, et al. A chromatin-mediated reversible drug-tolerant state in cancer cell subpopulations. Cell. 2010;141:69-80.

12. Helming KC, Wang X, Wilson BG, Vazquez F, Haswell JR, Manchester HE, et al. ARID1B is a specific vulnerability in ARID1A-mutant cancers. Nat Med. 2014;20:251-4.

13. Khursheed M, Kolla JN, Kotapalli V, Gupta N, Gowrishankar S, Uppin SG, et al. ARID1B, a member of the human SWI/SNF chromatin remodeling complex, exhibits tumour-suppressor activities in pancreatic cancer cell lines. $\mathrm{Br} \mathrm{J}$ Cancer. 2013;108:2056-62.

14. Denis GV, Green MR. A novel, mitogen-activated nuclear kinase is related to a Drosophila developmental regulator. Genes Dev. 1996;10:261-71.

15. Cheung KL, Zhang F, Jaganathan A, Sharma R, Zhang Q, Konuma $\mathrm{T}$, et al. Distinct roles of Brd2 and Brd4 in potentiating the transcriptional program for Th17 cell differentiation. Mol Cell. 2017;65:1068-80 e5.

16. Marcotte R, Sayad A, Brown KR, Sanchez-Garcia F, Reimand J, Haider M, et al. Functional genomic landscape of human breast cancer drivers, vulnerabilities, and resistance. Cell. 2016;164:293-309.

17. Bitler BG, Aird KM, Garipov A, Li H, Amatangelo M, Kossenkov AV, et al. Synthetic lethality by targeting EZH2 methyltransferase activity in ARID1A-mutated cancers. Nat Med. 2015;21:231-8.

18. Miller RE, Brough R, Bajrami I, Williamson CT, McDade S, Campbell J, et al. Synthetic lethal targeting of ARID1A-mutant ovarian clear cell tumors with dasatinib. Mol Cancer Ther. 2016;15:1472-84.
19. Bitler BG, Wu S, Park PH, Hai Y, Aird KM, Wang Y, et al. ARID1A-mutated ovarian cancers depend on HDAC6 activity. Nat Cell Biol. 2017;19:962-73.

20. Murakami R, Matsumura N, Brown JB, Higasa K, Tsutsumi T, Kamada M, et al. Exome sequencing landscape analysis in ovarian clear cell carcinoma shed light on key chromosomal regions and mutation gene networks. Am J Pathol. 2017;187:2246-58.

21. Anders S, Huber W. Differential expression analysis for sequence count data. Genome Biol. 2010;11:R106.

22. Luo B, Cheung HW, Subramanian A, Sharifnia T, Okamoto M, Yang X, et al. Highly parallel identification of essential genes in cancer cells. Proc Natl Acad Sci USA. 2008;105:20380-5.

23. Herold MJ, van den Brandt J, Seibler J, Reichardt HM. Inducible and reversible gene silencing by stable integration of an shRNAencoding lentivirus in transgenic rats. Proc Natl Acad Sci USA. 2008;105:18507-12.

24. Gad H, Koolmeister T, Jemth AS, Eshtad S, Jacques SA, Strom $\mathrm{CE}$, et al. MTH1 inhibition eradicates cancer by preventing sanitation of the dNTP pool. Nature . 2014;508:215-21.

25. Prahallad A, Heynen GJ, Germano G, Willems SM, Evers B, Vecchione L, et al. PTPN11 Is a Central Node in Intrinsic and Acquired Resistance to Targeted Cancer Drugs. Cell Rep. 2015;12:1978-85.

26. Alkema NG, Tomar T, Duiker EW, Jan Meersma G, Klip H, van der Zee AG, et al. Biobanking of patient and patient-derived xenograft ovarian tumour tissue: efficient preservation with low and high fetal calf serum based methods. Sci Rep. 2015;5:14495.

27. Frank SR, Schroeder M, Fernandez P, Taubert S, Amati B. Binding of c-Myc to chromatin mediates mitogen-induced acetylation of histone $\mathrm{H} 4$ and gene activation. Genes Dev. 2001;15:2069-82.

28. Delmore JE, Issa GC, Lemieux ME, Rahl PB, Shi J, Jacobs HM, et al. BET bromodomain inhibition as a therapeutic strategy to target c-Myc. Cell . 2011;146:904-17.

29. Shao Q, Kannan A, Lin Z, Stack BC Jr, Suen JY, Gao L. BET protein inhibitor JQ1 attenuates Myc-amplified MCC tumor growth in vivo. Cancer Res. 2014;74:7090-102. 